\title{
Analyzing Influence of the Transmission Medium on Timing Signals Transmitted by Hybrid Optical Transport Technologies
}

\author{
Rastislav Róka and Veronika Dolinayová
}

\begin{abstract}
The contribution deals with synchronization aspects in SDH and Synchronous Ethernet timing networks utilizing optical transmission medium. First, it explains a hierarchical configuration of timing network elements, characterizes transmission features of the optical fiber, particularly its main negative influences influencing transmitted information signals. The main part is dedicated to the evaluation program created for analyzing influences of the optical transmission path on timing signals transmitted by SDH and SyncE transport technologies. At last, resultant values from the introduced evaluation program are presented and evaluated.
\end{abstract}

Keywords - optical transmission medium, SDH and Synchronous Ethernet Technologies, timing networks, transmission parameters.

\section{INTRODUCTION}

For developing future services and applications [1], [2], solving of synchronizations for digital signals transmitting in telecommunication networks must be globally applicable and beneficial. Therefore, synchronization is one from key aspects at the signal transmission through metallic, optical or other transmission media by various different technologies [3], [4]. From the synchronization viewpoint, digital transmission networks can be divided into two groups - synchronous and asynchronous. In this contribution, we focus on timing signals transmitted by SDH (Synchronous Digital Hierarchy) and SyncE (Synchronous Ethernet) technologies utilizing the optical transmission medium.

For the signal transmission in SDH and Synchronous Ethernet networks, a dominating transmission medium is the optical fiber. It is a fact that various negative environmental effects influence any information or timing signals transmitted through the optical transmission medium. To the most important belong an attenuation

Manuscript received August 15, 2016, revised January 19, 2017

This article was created with the support of the Ministry of Education, Science, Research and Sport of the Slovak Republic within the KEGA agency project - 007STU-4/2016 "Progressive educational methods in the field of telecommunications multiservice networks" and VEGA agency project 1/0462/17 "Modeling of qualitative parameters in IMS networks".

Rastislav Róka is with Institute of MICT, FEI STU, Bratislava, Slovakia (corresponding author, phone: 421-2-68279608; e-mail: rastislav.roka@stuba.sk).

Veronika Dolinayová was with Institute of MICT, FEI STU, Bratislava, Slovakia (e-mail: veronika.dolinayova@gmail.com). doi: 10.11601/ijates.v6i1.186 occasioning the optical impulse's amplitude decreasing and a dispersion occasioning the optical impulse's time expansion. From a viewpoint of digital transmission technologies, these effects become expressively evident by changing of transmission parameters. Therefore, a maximum transmission rate in the optical medium is finite and a signal is transmitted to the definite transmission distance depending on the optical source, parameters of the optical fiber and the optical receiver at the optical transmission path's end. In this contribution, the aim is focusing on the optical transmission medium and its impact on transmission parameters of timing signals utilized by SDH and Synchronous Ethernet technologies. The created evaluation program can be utilized for evaluation of exploited optical transmission paths transmitting digital signals of future services and applications.

\section{SDH AND SYNCHRONOUS ETHERNET TIMING NETWORKS}

\section{A. SDH timing network}

A timing architecture in the $\mathrm{SDH}$ digital transmission network is hierarchical, thus every network node has its specific frequency, respectively timing accuracy. This architecture is splitted into 4 layers with strictly defined timing accuracies [5]. Individual SDH timing network elements include build-in frequency generators with the certain accuracy that is not absolutely identical for all elements. Deviations from the nominal synchronization frequency transmitted from node to node are summed and, in consequence, the crash of synchronization can come into the existence at the optical transmission path's end. From this reason, a common primary source of the timing signal is established with the highest Stratum 1 accuracy in the hierarchical structure [5].

A structure of timing elements in the SDH network is hierarchical, defined as the master-slave structure. A basic arrangement is the synchronizing chain, where at the highest level is presented by the PRC (Primary Reference Clock) clock. The second level below is formed by the SSU (Synchronization Supply Unit) clock. The SEC (Synchronous Equipment Clock) clock on the lowest level with the minimum accuracy demand is located in individual SDH timing network elements. According to ITU-T recommendations, the maximum number of elements coupled in the synchronizing chain is defined as 
follows: a maximum of 20 SEC elements located between $2 \mathrm{SSU}$ elements, a maximum of $10 \mathrm{SSU}$ elements and 59 SEC elements in the common chain [5], [6]. The ITU-T recommendation [7] describes the functional architecture of transport networks including network synchronization principles for networks that are based on the SDH.

\section{B. Synchronous Ethernet timing network}

The Synchronous Ethernet technology is defined in the ITU-T Q13/15 recommendation, where a distribution of timing signals in the SyncE network is specified. The reference timing signal from the PRC clock is distributed in the Synchronous Ethernet network by similar mechanisms as in the SDH network. Above all, a timing signal is separated from information, managing and controlling signals and then is transmitted through the physical layer [8].

The IETF (Internet Engineering Task Force) organization specified the CES (Circuit Emulation Service) element for interconnecting SDH and Synchronous Ethernet timing networks. For correct interoperability and cooperation between these two networks, the CES element is referred to be as the slave and must have the same synchronization features as the SEC one. A maximum number of CES elements in the synchronizing chain is defined 20 [9]. The ITU-T recommendation [7] also includes the application of various mappings.

\section{TRANSMISSION FEATURES OF THE OPTICAL TRANSMISSION PATH}

\section{A. Attenuation in the optical transmission medium}

The attenuation, alternatively the loss, is depending on the wavelength and is composed from scattering, absorption and bending parts. The total attenuation can be calculated using the formula [5]:

$$
a=\alpha \cdot L,
$$

where $a$ is the attenuation in $[\mathrm{dB}], \alpha$ is the specific attenuation in $[\mathrm{dB} / \mathrm{km}]$ and $L$ represents the optical fiber length in $[\mathrm{km}]$. This attenuation simultaneously corresponds to a difference between the transmitter power and the receiver sensitivity and, therefore, can be used for determination of the maximum transmission distance.

\section{B. Dispersion in the optical transmission medium}

The dispersion is composed from three main parts - modal, chromatic and polarization mode. The modal dispersion is due to various track lengths of particular modes and is present in multi-mode optical fibers. In single-mode fibers, the chromatic dispersion depending on the spectral bandwidth of optical sources becomes evident. In these fibers, the polarization mode dispersion is also originating and, therefore, a resultant value is given from contributions of both CD and PMD components [10]. Then, a final time expansion of the transmitted optical impulse is determined. For successful and reliable separation of particular optical impulses, a maximum transmission rate of the digital signal is limited by a following equation:

$$
R_{b}=\frac{1}{T_{b}}
$$

where $R_{b}$ is the transmission bit rate in [bit/s] and $T_{b}$ is the time duration of one information bit in [s]. For correct detecting of the information optical impulse, a maximum of its time expansion is theoretically limited to a half of the time duration of one information bit. For more real approximation, a possible impulse time expansion is set to the $1 / 10 \cdot T_{b}$ value in the program.

\section{Parameters of used components}

A optical transmission path utilizing the optical transmission medium is composed from following components:

- a source of the optical radiation,

- an optical fiber,

- a detector of the optical radiation.

Concrete values related to the source power, the specific attenuation and dispersion of the optical fiber and the receiver sensitivity used in the created evaluation program can be found in [11]-[16].

\section{THE EVALUATION PROGRAM}

The core of this contribution is an evaluation program for analyzing influences of the optical transmission medium on timing signals transmitted by hybrid transport technologies through evaluating transmission parameters. It is created by using the MatLab programming environment.

In timing networks, we can distinguish 4 basic types of the synchronization clock (Type I - IV) that are determined by their accuracy (in a time and/or frequency domain). These four synchronization clocks are organized in the hierarchical timing structure where the strongest requirements are defined for the highest level related to the PRC. The Primary Reference Clock represents the Type I clock. For this reason, it is satisfactory to focus our attention only on the Type I level. Other clocks located on lower levels are constrained with less rigorous demands. The Appendix III in [7] contains concrete guidelines for synchronization network engineering where all aspects related to the arrangement of synchronization clocks are explained by detail. For timing signals transmitted in the optical transmission medium, it is important to specify a concrete carrier wavelength of the optical radiation. For selecting adequate sources of the optical radiation, these specific source wavelengths together with their spectral bandwidth (deviations) are important.

For the Type I clock, a defined maximum deviation for the timing signal is $1,5 \mathrm{~nm}$ modulated at the source wavelength. Typical utilized source of the optical radiation in practical transmission systems has a broader defined minimum spectral bandwidth [11]. Because a bandwidth of the modulated timing signal is included in this minimum spectral bandwidth, the Type I clock timing signal is not necessary to adapt for transmitting through the optical medium. Hereby, it is satisfactory to consider only mentioned source spectral bandwidth in the evaluation 
program.

\section{A. Graphical interface}

After running the program, the opening screen is displayed (Fig. 1).

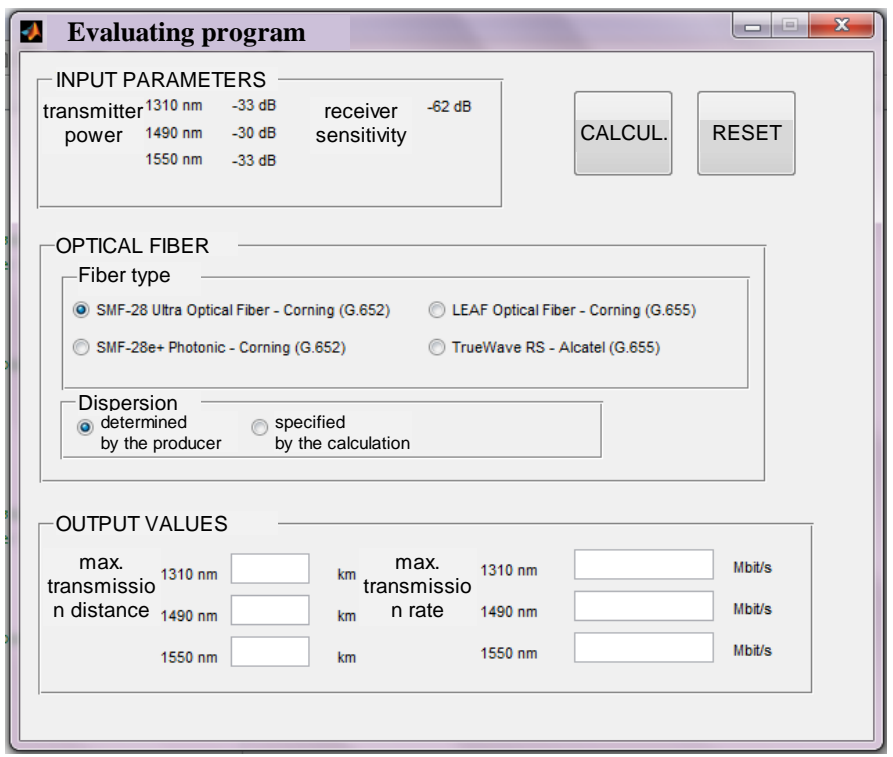

Fig. 1: The graphical interface of the evaluation program

In the first evaluation step, parameters for the source and the detector of the optical radiation are presented. Consequently, using input values of the transmitter power $P_{\text {tran }}$ in $[\mathrm{dB}]$ and the receiver sensitivity $S_{\text {rec }}$ in $[\mathrm{dB}]$ together with the specific attenuation value $\alpha_{O F}$ in $[\mathrm{dB} / \mathrm{km}]$ for the selected fiber type, a calculation can be executed for defined wavelengths. A maximum transmission distance $L$ of the optical path transmission can be calculated using a following formula resulting from the formula (1):

$$
L=\frac{1}{\alpha_{O F}}\left(10 \cdot \log \frac{P_{\text {trans }}}{S_{\text {rec }}}\right),
$$

In the evaluation program, considered optical fibers are presented. In the part "Fiber type", 4 considered kinds of optical fibers are included - two are based on the ITU-T G.652 standard [17] and two on the ITU-T G.655 standard [18]. Parameters of these fiber types are used in both evaluation steps by appropriate way. As a default choice, the Corning SMF-28 Ultra optical fiber and values determined by the producer are predetermined.

In the second evaluation step, an approach for dispersion calculating must be selected. There come into consideration two choices - concrete values determined by the producer or values considering the spectral bandwidth of the source. Using obtained values of the chromatic dispersion $D_{C H}$ in $[\mathrm{ps} /(\mathrm{nm} . \mathrm{km})]]$ for the selected fiber type and the source spectral bandwidth $B W_{\text {source }}$ in [nm] together with the calculated maximum transmission distance $L$, a calculation can be executed for defined wavelengths. A transmission rate $R_{b}$ of the optical information signal can be calculated using following formulas resulting from the formula (2):

$$
\begin{aligned}
\Delta t_{C H} & =\frac{D_{C H}}{B W_{\text {source }} \cdot L}, \\
R_{b} & =\frac{1}{10 \cdot \Delta t_{C H}},
\end{aligned}
$$

where $\Delta t_{C H}$ is the impulse time expansion in [ps] due to the chromatic expansion of optical fibers.

Finally, output values of the evaluation are presented. Here, the maximum transmission distance $L[\mathrm{~km}]$ of the optical radiation together with the maximum transmission rate $R_{b}[\mathrm{Mbit} / \mathrm{s}]$ of the information signal are displayed for particular source wavelengths.

\section{B. Resultant values for the maximum transmission distance and transmission rates}

Resultant values of the maximum transmission distance $L$ and transmission rates $R_{b \text { det }}$ and $R_{b \text { func }}$ for Corning SMF-28 Ultra and Corning SMF-28e+ Photonic optical fibers are introduced in Tables I and II. The transmission rate $R_{b \text { det }}$ is calculated based on values determined by the producer, the transmission rate $R_{b f u n c}$ is calculated from functionally dependent values on wavelengths for the specific attenuation and chromatic dispersion.

TABLE I

RESULTANT VALUES FOR THE CORNING SMF-28 ULTRA OPTICAL FIBER

\begin{tabular}{cccc}
\hline \hline$\lambda[\mathrm{nm}]$ & $L[\mathrm{~km}]$ & $R_{b \text { det }}[\mathrm{Mbit} / \mathrm{s}]$ & $R_{b \text { func }}[\mathrm{Mbit} / \mathrm{s}]$ \\
\hline 1310 & 82,8571 & 109858,8 & 18,3291 \\
1490 & 160,000 & 79056,9 & 7,4452 \\
1550 & 145,000 & 0,9576 & 0,8900 \\
\hline \hline
\end{tabular}

TABLE II

RESUlTANT VALUES FOR THE CORNING SMF-28E+ PHOTONIC OPTICAL FIBER

\begin{tabular}{cccc}
\hline \hline$\lambda[\mathrm{nm}]$ & $L[\mathrm{~km}]$ & $R_{b \text { det }}[\mathrm{Mbit} / \mathrm{s}]$ & $R_{b \text { func }}[\mathrm{Mbit} / \mathrm{s}]$ \\
\hline 1310 & 82,8571 & 109858,8 & 16,7498 \\
1490 & 133,333 & 86602,5 & 9,3439 \\
1550 & 145,000 & 0,9576 & 0,9687 \\
\hline \hline
\end{tabular}

The Corning SMF-28 Ultra and Photonic optical fibers correspond to the ITU-T G.652 standard [17]. They present classical single-mode fibers used in telecommunications. The zero value of the chromatic dispersion is located around the $1310 \mathrm{~nm}$ wavelength, where the maximum achievable rate $R_{b \text { det }}$ approximately $100 \mathrm{Gbit} / \mathrm{s}$ is resulting. When a spectral bandwidth of the optical source is considering together with a functional dependency on wavelengths for the chromatic dispersion, a theoretical maximum achievable rate $R_{b \text { func }}$ is decreased to tens of $\mathrm{Mbit} / \mathrm{s}$. A value of the chromatic dispersion determined by the producer at the $1550 \mathrm{~nm}$ wavelength is approximately the same as a functionally dependent value, therefore maximum achievable transmission rates are roughly equal for both cases.

A specific attenuation of the SMF-28 Ultra optical fiber has the lowest value at the $1490 \mathrm{~nm}$ wavelength. It means that the longest transmission distance $L$ can be reached 
exactly at this wavelength. For this case, however, a transmission rate is decreased due to the signal transmission over this distance. The lowest value of the specific attenuation for the SMF-28e+ Photonic optical fiber is present at the $1550 \mathrm{~nm}$ wavelength; hence a signal travels the longest transmission distance at this wavelength for given fiber.

Resultant values of the maximum transmission distance $L$ and transmission rates $R_{b \text { det }}$ and $R_{b \text { func }}$ for Corning LEAF and Alcatel Lucent TrueWave RS optical fibers are introduced in Tables III and IV.

TABLE III

RESULTANT VALUES FOR THE CORNING LEAF OPTICAL FIBER

\begin{tabular}{cccc}
\hline \hline$\lambda[\mathrm{nm}]$ & $L[\mathrm{~km}]$ & $R_{b \text { det }}[\mathrm{Mbit} / \mathrm{s}]$ & $R_{b \text { func }}[\mathrm{Mbit} / \mathrm{s}]$ \\
\hline 1310 & 85,2941 & 12,4349 & 11,0904 \\
1490 & 145,4545 & 23,2488 & 23,0484 \\
1550 & 138,0952 & 4,5259 & 2,9817 \\
\hline \hline
\end{tabular}

TABLE IV

RESUltaNT VALUES FOR THE ALCATEL LUCENT TRUEWAVE RS OPTICAL FIBER

\begin{tabular}{cccc}
\hline \hline$\lambda[\mathrm{nm}]$ & $L[\mathrm{~km}]$ & $R_{b \text { det }}[\mathrm{Mbit} / \mathrm{s}]$ & $R_{b \text { func }}[\mathrm{Mbit} / \mathrm{s}]$ \\
\hline 1310 & 72,5000 & 3,8314 & 3,3642 \\
1490 & 106,6667 & 69,4444 & 64,1026 \\
1550 & 116,0000 & 3,5920 & 2,9727 \\
\hline \hline
\end{tabular}

The Corning LEAF and Alcatel Lucent TrueWave RS optical fibers correspond to the ITU-T G.655 standard [18]. They present optical fibers with the shifted zero value of the chromatic dispersion to the $1550 \mathrm{~nm}$ wavelength surroundings. For both kinds, the producer determined a functional dependency of the chromatic dispersion on wavelengths. Therefore, values of the maximum theoretical transmission rates are approximately equal in both cases. Both optical fibers reach the maximum transmission rates around the $1490 \mathrm{~nm}$ wavelength. The TrueWave RS optical fiber reaches higher transmission rate at this wavelength, however a signal travels shorter transmission distances compared with the LEAF optical fibers. From calculated values, we can consider that the TrueWave RS directional vector forms a bigger angle with the horizontal axis than the LEAF one for about linear functional dependencies on wavelengths for the chromatic dispersion.

In practice, results from the evaluation program can be used in different ways, for instance at a selection of the wavelength for the optical channel with given transmission rates, respectively at a determination of the transmission rate for selected wavelengths. Just as well, a specifying of the source working wavelength for the longest transmission distance on the optical fiber's parameters can be realized based on presented resultant values. Globally, the presented evaluation program with analyzing of specific optical fibers can be utilized for evaluation of exploited optical transmission paths transmitting digital signals. In consequence, applicability and benefits of the optical transmission medium for transmitting timing signals for developed future services and applications and global telecommunication networks can be determined.

\section{CONCLUSION}

The program for analyzing influences of the optical transmission medium on timing signals transmitted by hybrid transport technologies through evaluating transmission parameters is created and related to a synchronization in SDH and Synchronous Ethernet timing networks.

A timing signal travelling through the optical fiber is influenced by various negative environmental effects. To the most expressive ones belong an attenuation and a dispersion, therefore it is important to know exact values for these parameters and/or exact functional dependencies on wavelengths for given parameters. From this reason, a timing signal is travelling through the optical transmission path with a finite transmission rate on a definite transmission distance. Results from the evaluation program prove that there can be found marked differences based on approaches to the dispersion characteristics for some types of optical fibers. These differences can lead to significant problems at the practical realization of multiple optical channel transmissions in hybrid transport systems using a wavelength division multiplexing technique.

The created evaluation program satisfies essential demands for determination a maximum transmission distance and a maximum transmission rate of timing signals transmitted through the optical transmission path. The program is created in the MatLab programming environment with a simple and comprehensible graphical interface. In a near future, possibilities for selecting of source, optical fibers and detectors can be easily extended. Moreover, other optical components (namely connectors, couplers, welds, ...) utilized in conjunction with the optical fiber can be included for analyzing influences of the optical transmission medium on timing signals.

In the next phase, an influence of network nodes included in the optical transmission path will be analyzed. For this intention, two direction of research are considered. First, network nodes realized with electro-optical and optoelectrical conversions will be supposed. Second, network nodes with only optical processing and controlling of timing signals are assumed.

\section{REFERENCES}

[1] M. Kellovský, I. Baroňák， M. Kavacký, E. Chromý, “The Optimal Sizing of HSS Database in IMS", Wireless Personal Communications, vol. 86, 2016, pp. 1-14, doi:10.1007/s11277-016-3750-6.

[2] J. Sitárová, M. Maár, M. Orgoň, "The Enterprise Telecommunication Network Design and its Implementation Using Technology PLC", International Journal on Recent and Innovation Trends in Computing and Communication, vol. 4, 2016, pp. 95-104.

[3] S. Klúčik, E. Chromý, I. Baroňák, "Model to increase the number of output sates of a random variable using a histogram based PDF", Wireless Personal Communications, vol. 85, 2015, pp. 137-149, doi:10.1007/s1 1277-015-2731-5.

[4] M. Nízky, M. Orgoň, "Mobile Antenna Inetvu 1800+ Series and Its Implementation in Practice", International Journal of Wireless and Microwave Technologies, January 2016, doi:10.5815/ijwmt.

[5] V.K. Stamarions, Understanding SONET/SDH and ATM Communications Networks for the Next Millennium. Lucent Technologies, USA, 1999, ISBN 0-7803-4745-5. 
[6] J. Ferrant, SDH Synchronization. London, United Kingdom, October 2005, available: http://www.scribd.com/doc/57003844/SDHSynchronization.

[7] Architecture of transport networks based on the synchronous digital hierarchy (SDH), ITU-T Recommendation G.803, 2000.

[8] A. Magree, "Synchronization in Next-Generation Mobile Backhaul Networks“, IEEE Comm. Magazine, vol. 48, 2010, pp. 110 - 116.

[9] Z. Ghebretensaé, J. Harmatos, K. Gustafsson, "Mobile Broadband Backhaul Network Migration from TDM to Carrier Ethernet", IEEE Communications Magazine, vol. 48, 2010, pp. 102 - 109.

[10] J. Čuchran, R. Róka, Optocommunication Systems and Networks. STU Publishing House, Bratislava, 2000, ISBN 80-227-2437-8.

[11] ThorLabs. Coherent Sources-Laser Diodes by Wavelength. available (20/10/2016): https://www.thorlabs.com/navigation.cfm

[12] Corning SMF-28 Ultra Optical Fiber. available (20/10/2016): https://www.corning.com/media/worldwide/coc/documents/Fiber/SMF28 Ultra.pdf

[13] Corning SMF-28e+ Photonic Optical Fiber. available (20/10/2016): http://www.corning.com/media/worldwide/csm/documents/Photonic Fiber_040113_uploaded 12_15_15.pdf

[14] Corning LEAF Optical Fiber. available (20/10/2016): http://www.corning.com/media/worldwide/coc/documents/Fiber/PI1107 07-14 English.pdf

[15] Lucent TrueWave $R S$ - Nonzero-dispersion optical fiber. available (20/10/2016):

http://www.telepp.com/support/broadband coax/truewave/true wave.pd $\underline{\mathrm{f}}$

[16] OSI LaserDiode. InGaAs APD/PIN Modules. available (20/10/2016): http://www.laserdiode.com/standard_products.

[17] Characteristics of a single-mode optical fiber and cable, ITU-T Recommendation G.652, 2009.

[18] Characteristics of a non-zero dispersion-shifted single-mode optical fiber and cable, ITU-T Recommendation G.655, 2009.
Rastislav Róka was born in Sal'a, Slovakia on January 27, 1972. He received his M.Sc. and Ph.D. degrees in Telecommunications from the Slovak University of Technology, Bratislava, in 1995 and 2002. Since 1997, he has been working as a senior lecturer at the Institute of Telecommunications, Faculty of Electrical Engineering and Information Technology, Slovak University of Technology in Bratislava. Since 2009, he is working as an associated professor at this institute.

His research and educational activities are focused on the signal transmission realized in fixed transmission media, optocommunication transmission systems and networks. A main effort is dedicated to effective utilization of the optical fiber's transmission capacity by using various optical signal processing techniques and dynamic bandwidth and wavelength allocation algorithms.

Veronika Dolinayová was born in Žilina, Slovakia on October 10, 1992. She received her M.Sc. degree in Telecommunications from the Slovak University of Technology, Bratislava, in 2016. 